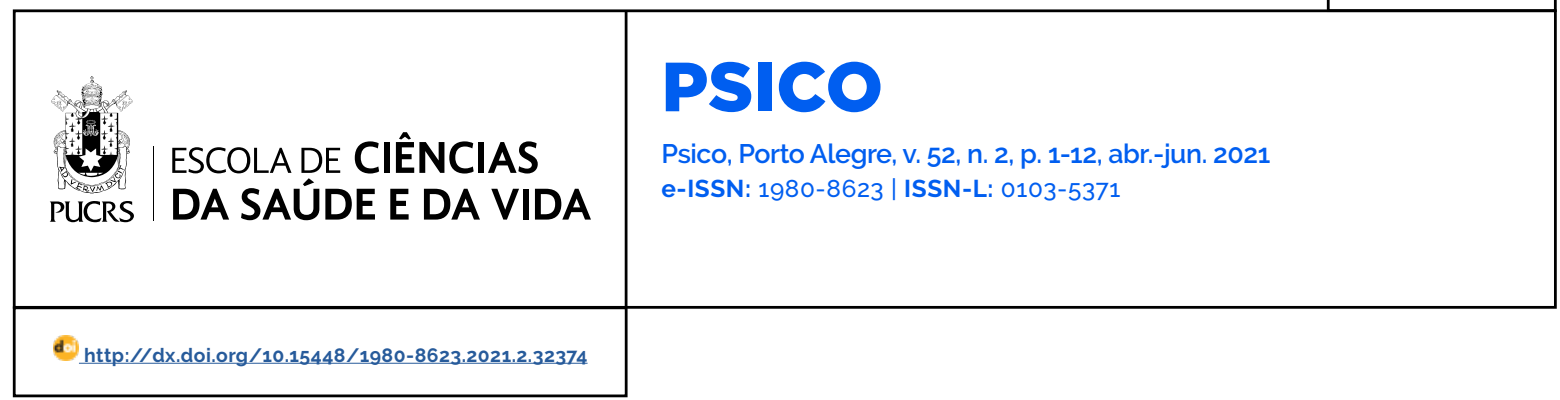

SEÇÃO: ARTIGO

\title{
Habilidades sociais e expectativas de futuro como preditores da autoeficácia para a escolha profissional
}

\author{
Profissional social skills and future expectations as predictors of professional choice \\ self-efficacy \\ Habilidades sociales y expectativas de futuro como preditores de la autoeficacia para \\ la elección profesional
}

\section{Gisele Aparecida de \\ Morais ${ }^{1}$}

orcid.org/0000-0002-1640-7783

gisele.morais@iff.edu.br

\section{Vanessa Barbosa \\ Romera Leme ${ }^{2}$ \\ orcid.org/0000-0002-9721-0439 \\ vanessaromera@gmail.com}

\section{Amanda Oliveira Falcão \\ Medeiros $^{3}$}

orcid.org/0000-0003-1492-3431 amanda.falcao88@gmail.com

\section{Camila de Sousa \\ Pereira-Guizzo 4}

orcid.org/0000-0003-4197-8534 camila.pereira@fieb.org.br

Recebido em: 30 out. 2018 Aprovado em: 24 mar. 2021. Publicado em: 10 ago. 2021.
Resumo: Durante o ensino médio, os estudantes podem enfrentar as primeiras escolhas relacionadas ao seu futuro profissional. O estudo investigou em que medida o sexo, as habilidades sociais e as expectativas de futuro predizem a autoeficácia para a escolha profissional de estudantes do ensino médio. Participaram 436 alunos $(M=16,23$ anos, $D P=1,09)$, de ambos os sexos, de escolas públicas e privadas do estado do Rio de Janeiro. Os instrumentos utilizados foram: Escala de Autoeficácia para a Escolha Profissional; Escala de Expectativas Futuras; Inventário de Habilidades Sociais para Adolescentes; e Questionário de Dados Socioeconômicos. As habilidades sociais de desenvoltura social e abordagem afetiva, as expectativas de futuro em relação ao trabalho e à educação e o sexo feminino explicaram $26 \%$ da variabilidade da autoeficácia para a escolha profissional dos estudantes. Os dados da pesquisa poderão contribuir para o planejamento de futuros programas de intervenção com os alunos, seus familiares e professores. Palavras-chave: autoeficácia, escolha profissional, habilidades sociais

Abstract: During high school, students can face the first choices related to their professional future. The study investigated the extent to which gender, social skills and future expectations predict professional choice self-efficacy of high school students. A total of 436 students $(M=16,23, S D=1,09$ ) from both sexes from public and private schools in the State of Rio de Janeiro participated. The instruments used were: Professional Choice Self-efficacy Scale; Future Expectations Scale; Social Skills Inventory for Adolescents; Socioeconomic Status Questionnaire. Social skills of social resourcefulness and affective approach, expectations of the future in relation to work and education, and the female sex explained $26 \%$ of the variability of professional choice self-efficacy of students. The research data may contribute to the planning of future intervention programs with students, their families and teachers. Keywords: self-efficacy, occupational choice, social skills

Resumen: Durante la escuela secundaria, los estudiantes pueden enfrentar las primeras opciones relacionadas con su futuro profesional. El estudio investigó hasta qué punto el género, las habilidades sociales y las expectativas del futuro predicen la autoeficacia para la elección profesional de los estudiantes de secundaria. Participaron 436 alumnos $(M=16,23, D P=1,09)$, de ambos sexos de escuelas públicas y particulares en el Estado de Río de Janeiro. Los instrumentos utilizados fueron: Escala de Autoeficacia para la elección profesional; Escala de Expectativas Futuras; Inventario de Habilidades Sociales para Adolescentes; Cuestionario de datos socioeconómicos. Las habilidades sociales de desenvoltura social y abordaje afectivo, las expectativas de futuro en relación al trabajo ya la educación y sexo femenino explicaron el $26 \%$ de la variabilidad 
de la autoeficacia para la elección profesional de los estudiantes. Los datos de la investigación pueden contribuir a la planificación de futuros programas de intervención con estudiantes, sus familias y maestros. Palabras clave: autoeficacia, elección profesional, habilidades sociales

A adolescência compreende, além das alterações biológicas, (por exemplo, a produção de hormônios de crescimento que desencadeia transformações nos órgãos sexuais) mudanças relacionadas ao contexto sociocultural e ao momento histórico, sendo uma fase do desenvolvimento humano importante na construção da identidade. Assim, as transformações biopsicossociais, tais como o amadurecimento sexual e a aquisição de maior autonomia e responsabilidade vivenciadas nesse período de vida, influenciam a forma como os adolescentes se percebem e se relacionam com seus pares, professores e familiares (Berni \& Roso, 2014). Além disso, resultam no reconhecimento do indivíduo como adulto por parte da sociedade, sendo esse status fortalecido e mantido pela execução de determinadas tarefas desenvolvimentais que comumente são associadas à vida adulta, como a escolha profissional, o casamento e a parentalidade e que demarcam o findar da adolescência e o ingresso na adultez (Ponciano \& Féres-Carneiro, 2014). Contudo, mudanças sociais e culturais que ocorreram nas sociedades pós-industriais a partir das últimas décadas do século passado, tais como o prolongamento dos estudos e as dificuldades de inserção no mercado de trabalho culminaram em um aumento da idade média do primeiro casamento e nascimento dos filhos, na entrada tardia no mercado de trabalho e no aumento no periodo de residência na casa dos pais. Desse modo, tem ocorrido um adiamento da entrada na vida adulta, marcada por extenso periodo de experimentações de diferentes escolhas em aspectos profissionais e pessoais pelos adolescentes, especialmente para os que têm alto nivel socioeconômico (Arnett, 2016; Dutra-Thomé \& Koller, 2017).

A escolha profissional é uma tarefa desenvolvimental importante e ocorre, via de regra, no final dos anos de ensino médio. Nesse período, os adolescentes se deparam com a tomada de de- cisão em relação ao futuro profissional, tendo que escolher por um curso superior, profissionalizante ou mesmo um emprego (Braga \& Xavier, 2016; Dantas \& Azzi, 2018; Noronha et al.,2013). Todavia, a percepção negativa desse momento devido à indecisão profissional, descrença nas capacidades pessoais, baixos recursos de enfrentamento, falta de autoconhecimento e de autoeficácia para coleta de informações profissionais, pode causar um adoecimento fisico e mental nos adolescentes (Noronha et al., 2013; Prisco et al., 2013). Diante disso, pesquisadores têm procurado investigar fatores motivacionais relacionados à escolha profissional na transição para a vida adulta, com destaque para as crenças de autoeficácia (Ambiel \& Noronha, 2012; Prisco et al., 2013).

A autoeficácia é um dos conceitos-chave da Teoria Social Cognitiva (TSC), proposta por Bandura (1986). A TSC adota o modelo de reciprocidade triádica que compreende o desenvolvimento do individuo como sendo influenciado pela interação reciproca de fatores pessoais, comportamentais e ambientais (Azzi et al., 2014; Bandura, 1989). As crenças de autoeficácia (fator pessoal) referem-se à confiança na capacidade pessoal para organizar e realizar ações especificas para alcançar determinados objetivos (Bandura, 1989). Essas crenças contribuem para a motivação humana, são associadas com bom desempenho escolar e menores niveis de problemas socioemocionais na transição para a vida adulta. As crenças de autoeficácia têm sido investigadas tanto de maneira ampla, como a autoeficácia geral, quanto de modo específico, como na escolha profissional de adolescentes e jovens (Ambiel \& Noronha, 2012; Dantas \& Azzi, 2018; Moreira et al., 2018; Prisco et al., 2013).

A autoeficácia para a escolha profissional refere-se à confiança que uma pessoa tem na sua capacidade em se envolver em atividades relacionadas à decisão de uma carreira (Ambiel \& Noronho, 2012; Taylor \& Betz, 1981). Diz respeito, portanto, às crenças nas possibilidades de tomar iniciativas e dar seguimento em tarefas relativas à escolha de uma profissão (Ambiel \& Noronha, 2012; Moreira et al., 2018). Há uma longa tradição de estudos internacionais, principalmente nor- 
te-americanos, que se propuseram a relacionar o construto da autoeficácia de Bandura (1989) com a escolha profissional (Ambiel \& Noronha, 2012; Hackett \& Betz, 1981; Taylor \& Betz, 1981). Dentre essas pesquisas, destaca-se a Teoria Social Cognitiva de Desenvolvimento de Carreira (TSCD), proposta por Lent et al. (1994), que enfatiza o papel das crenças de autoeficácia na elaboração de interesses, nas escolhas e no desempenho em atividades acadêmicas e profissionais. No âmbito internacional, a autoeficácia para a escolha profissional, que é a tradução de Career Decision-Making Self-Efficay, realizada por Ambiel e Noronha (2012), tem sido investigada em relação a diferentes aspectos, tais como estilos parentais e de apego, papeis de gênero e de ansiedade frente à escolha da carreira de estudantes (Bolat \& Odaci, 2017; Chiesa et al., 2016; Sovet \& Metz, 2014).

No contexto nacional, pesquisas têm identificado que adolescentes com niveis elevados de autoeficácia para a escolha profissional têm mais iniciativa, autoestima, autoconfiança frente às tarefas dificeis, objetivos mais claros, maior comprometimento e engajamento nas atividades que se propõem a fazer e tendência para liderar (Ambiel \& Noronha, 2012). Depreende-se disso que indivíduos com niveis elevados de autoeficácia para a escolha profissional apresentam maior chance de se envolver intensivamente em atividades relacionadas à escolha da profissão, direcionando suas ações para coleta de informações, autoconhecimento e autorrealização (Dantas \& Azzi, 2018).

Algumas pesquisas também sugerem que 0 sexo pode interferir no desenvolvimento das crenças de autoeficácia para escolha profissional. Estudos encontraram que as meninas apresentam mais crenças de autoeficácia para a escolha profissional (Ambiel \& Noronha, 2014; Guerreiro-Casanova et al., 2015: Noronha et al., 2013). Esse fato parece estar relacionado às mudanças nos papeis de gênero decorrentes de alterações socioeconômicas e culturais ocorridas nas últimas décadas, como a maior inserção das mulheres no mercado de trabalho e maior engajamento com a educação (Oliveira et al., 2016). Outras variáveis também impactam nas crenças de autoeficácia para a escolha profissional, tais como o suporte familiar, as práticas educativas parentais, o nivel socioeconômico, o lócus de controle e as competências interpessoais (Ambiel \& Noronha, 2012; Ventura \& Noronha, 2014).

No que se refere ao campo das relações interpessoais, as habilidades sociais compreendem comportamentos necessários a uma relação interpessoal bem-sucedida, conforme características da pessoa, situação, cultura e momento histórico, proporcionando relacionamentos satisfatórios e produtivos com as pessoas próximas (Del Prette \& Del Prette, 2010). Nesse sentido, supõe-se que as habilidades sociais podem impactar positivamente o desenvolvimento ou o fortalecimento das crenças de autoeficácia, considerando que as experiências pessoais são a maior fonte de influência para o desenvolvimento dessas crenças (Bandura, 2006). Na medida em que os adolescentes ampliam o repertório de habilidades sociais, eles podem vivenciar experiências de sucesso, aumentando, assim, as possibilidades de êxito nas tarefas futuras, o que contribui para aumentar ou fortalecer suas crenças de autoeficácia. Sustentando essa afirmação, pesquisas empíricas evidenciaram associações positivas entre as habilidades sociais e indicadores de autoeficácia (Erozkan, 2013; Harrel et al., 2009; Leme et al., 2016). Entretanto, nenhuma dessas investigações analisou conjuntamente as habilidades sociais e as crenças de autoeficácia para a escolha profissional, sugerindo uma lacuna nessa área de investigação.

Apesar das expectativas de futuro estarem presentes no decorrer de toda vida, influenciando o comportamento do indivíduo, pensamentos e planejamentos para o futuro são particularmente importantes na transição da adolescência para a vida adulta (Bandura, 1989; Nurmi, 1991). As expectativas de futuro são compreendidas como a antecipação de objetivos futuros por meio de um processo motivacional que integra o futuro cronológico ao presente da pessoa, gerando a crença de que aquilo que é almejado poderá acontecer (Husman \& Lens, 1999). Pesquisas têm evidenciado que estudantes com mais expectativas positivas 
com relação ao futuro tendem a se engajar mais em atividades que conduzem à profissão desejada (Walker \& Tracey, 2012; Jung et al., 2015). Portanto, especialmente na adolescência, as expectativas de futuro podem funcionar como um importante fator de proteção para o desenvolvimento psicossocial saudável (Jung et al., 2015; Dutra-Thomé et al., 2015), tendo em vista que a reflexão acerca do futuro motiva o comportamento atual, interferindo nas decisões, buscas e tarefas que podem afetar o alcance dos objetivos para o futuro (Beal \& Crockett, 2010; Nurmi, 1991).

Na literatura foram identificados três estudos internacionais (Jung et al., 2015; Tsuzuki, 2015; Walker \& Tracey, 2012) que investigaram a relação entre expectativas de futuro e de autoeficácia para a escolha profissional. Todavia, apenas o estudo de Tsuzuki (2015) foi realizado com uma amostra estudantes do ensino médio. Os outros estudos focalizaram o contexto do ensino superior (Jung et al., 2015; Walker \& Tracey, 2012). Esses dados indicam uma escassez de pesquisas e, consequentemente, a necessidade de se investigar conjuntamente essas variáveis, de forma a se obter maior conhecimento a respeito dos preditores da autoeficácia para a escolha profissional.

A decisão de seguir determinada profissão compreende um processo mais abrangente com implicações em aspectos interpessoais e emocionais na transição para a vida adulta e, de modo geral, ao longo de todo o curso de vida (Ambiel \& Noronha, 2012). Por isso, a inserção dos adolescentes na vida laboral tem sido tema na agenda psicológica em pesquisas descritivas (Prisco et al., 2013), na construção de instrumentos (Moreira et al., 2018) e na implementação de intervenções com estudantes do ensino médio (Dantas \& Ambiel, 2018). Como o trabalho é cada vez mais valorizado como uma fonte de realização pessoal, há um apelo ou pressão para que os adolescentes decidam quanto à carreira, tornando, muitas vezes, esse momento angustiante e conflitivo (Braga \& Xavier, 2016). Somado a isso, dados do último Censo Escolar da Educação Básica, realizado pelo Instituto Nacional de Estudos e Pesquisas Educacionais Anísio Teixeira
(Inep, 2017) revelaram que a evasão escolar é de $11,2 \%$ do total dos alunos do ensino médio, sinalizando altas taxas entre anos de 2014 e 2015. Dessa forma, torna-se relevante favorecer o desenvolvimento de recursos pessoais que possibilitem aos adolescentes perceberem as instituições escolares como um ambiente de oportunidades e que as experiências ali vivenciadas possam fomentar perspectivas positivas em relação ao futuro profissional, nas mais diversas áreas, contribuindo para a conclusão do ensino médio e o ingresso no ensino superior.

Contudo, apesar da constatação dos benefícios do desenvolvimento das crenças de autoeficácia para a escolha profissional, das habilidades sociais e de expectativas positivas com relação ao futuro dos adolescentes, faltam estudos no contexto brasileiro que investiguem essas variáveis conjuntamente com estudantes do ensino médio. A maior compreensão sobre a relação entre esses construtos pode fomentar intervenções preventivas e para a promoção de saúde mental com os adolescentes durante a transição para a vida adulta. Diante disso, este estudo procurou investigar em que medida o sexo, as habilidades sociais e as expectativas de futuro predizem a autoeficácia para a escolha profissional de estudantes do ensino médio.

\section{Método}

\section{Delineamento}

Estudo de caráter descritivo, correlacional e preditivo.

\section{Participantes}

Participaram deste estudo 436 estudantes, com idades entre 14 e 20 anos $(M=16,23, D P$ = 1,09), sendo 248 meninas e 188 meninos. Os participantes frequentavam o $1^{\circ}(n=163)$, o $2^{\circ}$ $(n=123)$ e o $3 .^{\circ}$ ano $(n=150)$ do ensino médio e eram provenientes de cinco escolas, sendo duas públicas e três particulares, do estado do Rio de Janeiro. Em relação à escolaridade dos pais ou cuidadores dos alunos, 22 (5,0\%) tinha nivel analfabeto/fundamental I incompleto; 48 $(11,0 \%)$ fundamental I completo/fundamental II 
incompleto, 80 (18,3\%) fundamental II completo/ médio incompleto, 139 (31,9\%) médio completo/ superior incompleto e 147 (33.7\%) superior completo. A amostra foi selecionada por conveniência.

\section{Instrumentos}

\section{Escala de Autoeficácia para a Escolha Profis-} sional (EAE-EP). Desenvolvida por Ambiel e Noronha (2014) com o objetivo de avaliar as crenças das pessoas na própria capacidade de se engajar em tarefas relativas à escolha profissional. $O$ instrumento contém 47 itens em que as respostas estão dispostas em uma escala tipo Likert de quatro pontos, variando de pouco (1) a muito (4). A escala apresenta quatro dimensões, com os seguintes índices de confiabilidade no estudo de validação: (1) Autoeficácia para autoavaliação ( $\alpha$ = 0,88, "Escolher uma profissão que você realmente goste"); (2) Autoeficácia para coleta de informações ocupacionais ( $\alpha=0,84$, "Fazer cursos após o ensino médio"); (3) Autoeficácia para busca de informação profissional prática ( $\alpha=0,84$, "Conversar com uma pessoa que trabalhe numa profissão pela qual você se interesse"); (4) autoeficácia para planejamento de futuro ( $\alpha=0,79$, "Informar-se a respeito do retorno financeiro que a profissão pode lhe trazer"); escore total $(\alpha=0,94)$. No presente estudo, foram encontrados os seguintes valores de consistência interna: autoeficácia para autoavaliação $(\alpha=0,82)$; autoeficácia para coleta de informações ocupacionais ( $\alpha=0,78)$; autoeficácia para busca de informação profissional prática ( $\alpha$ = 0,80); autoeficácia para planejamento de futuro $(\alpha=0,72)$; Total EAE-EP $(\alpha=0,90)$.

Inventário de Habilidades Sociais para Adolescentes (IHSA-Del-Prette). Desenvolvido por Del Prette e Del Prette (2009) para avaliar as habilidades sociais de adolescentes a partir dos seus autorrelatos sobre situações cotidianas. A versão reavaliada por Leme et al. (2016) é composta por 36 itens com respostas distribuidas em uma escala tipo Likert de cinco pontos, variando de nunca (0) a sempre (4). O inventário é formado por seis fatores, que no estudo de Leme et al. (2016) obtiveram os seguintes índices de consistência interna (Alfa de Cronbach): (1) Empatia ( $\alpha=0,80$, "Quando um amigo tem uma posição contrária à minha, consigo negociar uma solução boa para nós dois"); (2) Autocontrole ( $\alpha=0,73$, "Ao ser injustamente criticado, consigo responder sem perder o controle"); (3) Civilidade ( $\alpha=0,75$, "Ao sair de um local, eu me despeço das pessoas"); (4) Assertividade ( $\alpha=0,63$, "Consigo tomar a iniciativa de encerrar a conversa (bate-papo) com outra pessoa"); (5) Abordagem afetiva ( $\alpha=0,68$, "Quando estou a fim de ficar com alguma pessoa, eu digo isso a ele (a) na primeira oportunidade"); (6) Desenvoltura social ( $\alpha=0,61$, "Nos trabalhos de grupo, explico as tarefas aos colegas quando necessário"). No presente estudo, foram encontrados os seguintes valores de consistência interna: presente Empatia $(\alpha=0,69)$; Autocontrole $(\alpha=0,74)$; Civilidade $(\alpha=$ $0,66)$; Assertividade $(\alpha=0,52)$; Abordagem afetiva $(\alpha=0,53)$; Desenvoltura social $(\alpha=0,50)$

Escala de Expectativas Futuras para Adolescentes (FESA). Desenvolvida por McWhirter e McWhirter (2008) com estudantes chilenos e validada para o Brasil por Dutra-Thomé et al. (2015). O instrumento tem como objetivo conhecer expectativas de adolescentes com relação ao futuro em domínios específicos, como trabalho, educação, familia e religião. O instrumento é composto por 22 itens com respostas dispostas em uma escala tipo Likert de cinco pontos, variando de definitivamente não (1) a definitivamente sim (5), que procuram identificar o quanto os adolescentes gostariam que as situações descritas acontecessem no futuro. A escala é subdividida em seis fatores conforme os seguintes indices de confiabilidade encontrados por Dutra-Thomé et al. (2015): Trabalho e educação ( $\alpha=0,85$, "Alcançarei um nível de educação que eu quero"; Igreja ( $\alpha=0,73$, "Irei à missa ou a outros serviços religiosos regularmente"; Casamento $(\alpha=0,73$, "Meu casamento durará para sempre"; Saúde ( $\alpha=$ 0,64, "Terei uma alimentação saudável"; Crianças e familia ( $\alpha=0,69$, "Darei a meus filhos um lugar seguro para viver"). No presente estudo foram encontrados os seguintes valores de consistência interna: Trabalho e educação $(\alpha=0,80)$; Crianças e familia $(\alpha=0,73)$; Casamento $(\alpha=0,76)$; Igreja ( $\alpha$ $=0,89) ;$ Saúde $(\alpha=0,69)$. 


\section{Questionário com informações sociodemo-}

gráficas. O questionário com informações demográficas foi elaborado por este estudo a fim de investigar as caracteristicas sociais e demográficas dos participantes e suas familias.

\section{Procedimentos de coleta de dados}

A pesquisadora entrou em contato pessoalmente com as escolas eleitas por conveniência, para apresentar o projeto de pesquisa e pedir permissão às diretoras para a coleta de dados junto aos alunos. Em seguida, agendou com os professores um horário para a apresentação da pesquisa aos alunos e, nessa oportunidade, foram esclarecidos os objetivos da pesquisa, os procedimentos de coleta de dados e entregues duas cópias do Termo de Consentimento Livre e Esclarecido (TCLE) e do Termo de Assentimento (TA). Após a entrega dos termos, assinados pelos responsáveis legais dos alunos e pelos próprios estudantes, respectivamente, a coleta de dados, que ocorreu da mesma maneira nas cinco instituições, foi realizada de forma coletiva nas salas de aula dos estudantes, no horário que foi previamente combinado com os professores, com duração média de 50 minutos. Todos os alunos convidados e seus responsáveis aceitaram participar do estudo, sendo 284 estudantes de duas escolas públicas e 152 de três escolas particulares. A coleta de dados teve duração de três meses e contou com a participação da primeira autora e de outra psicóloga que auxiliou na entrega e no recolhimento dos instrumentos. A pesquisa, condizente com a Resolução 466/2012 do Conselho Nacional de Saúde, foi aprovada pelo Comitê de Ética em Pesquisa da Universidade Salgado de Oliveira (UNIVERSO-Niterói), parecer número 1.459.039 (CAAE 53947515.9.0000.5289).

\section{Procedimentos de análise de dados}

A análise de dados foi executada com o software Statistical Package for the Social Sciences for Windows (SPSS, versão 22.0). Primeiramente, foram testados e confirmados os pressupostos de normalidade. Posteriormente, calculou-se a correlação entre as variáveis (coeficiente $r$ de Pearson). Para interpretar a magnitude dos coeficientes de correlação foram adotados os seguintes valores: $<0,20=$ muito baixa; $>0,20$ e $<0,40=$ baixa; $>0,40$ e $<0,70=$ moderada; $>$ 0,70 e $<0,90=$ alta; $>0,90=$ muito alta (Bryman \& Cramer, 2003). Na sequência, foram testados e confirmados os pressupostos independência e multicolinearidade para o modelo preditivo e, em seguida, procedeu-se a análise de regressão hierárquica (método enter). O escore geral autoeficácia para a escolha profissional foi a variável de desfecho no modelo. No Bloco 1 foi inserida a variável sexo. No Bloco 2 foram incluidos os fatores das habilidades sociais empatia, autocontrole, civilidade, assertividade, abordagem afetiva e desenvoltura social. No Bloco 3 foram inseridos os fatores da expectativa de futuro trabalho e educação, crianças e família, casamento, igreja e saúde. Foi criada variável dummy em substituição à variável independente não métrica sexo $(1=$ feminino; 0 = masculino) .

\section{Resultados}

A Tabela 1 apresenta as correlações entre as variáveis (fatores e total) autoeficácia para a escoIha profissional, habilidades sociais e expectativas de futuro. Identificou-se associação significativa, positiva e de baixa magnitude entre habilidade sociais, expectativas de futuro e autoeficácia para escolha profissional. 
Tabela 1 - Correlações entre a Autoeficácia para a Escolha Profissional e Habilidades Sociais e Expectativas Futuras

\begin{tabular}{|c|c|c|}
\hline Variáveis & $M(D P)$ & Autoeficácia para a escolha profissional \\
\hline \multicolumn{3}{|l|}{ Habilidades Sociais } \\
\hline Empatia & $29,10(5,88)$ & $0,30^{\prime \prime}$ \\
\hline Autocontrole & $16,27(6,10)$ & 0,01 \\
\hline Assertividade & $20,52(4.53)$ & 0,22 \\
\hline Desenvoltura social & $12,78(3,72)$ & 0,39 \\
\hline Civilidade & $16,13(3,30)$ & 0,21 \\
\hline Abordagem afetiva & $9,04(3,93)$ & 0,21 \\
\hline Total & $103,75(18,06)$ & $0,33^{\prime \prime}$ \\
\hline \multicolumn{3}{|l|}{ Expectativas Futuras } \\
\hline Trabalho e educação & $30,60(3,43)$ & 0,33 \\
\hline Crianças e familia & $21,61(2,74)$ & $0,11^{\circ}$ \\
\hline Casamento & $11,08(2,63)$ & 0,04 \\
\hline Igreja & $10,47(3,61)$ & 0,07 \\
\hline Saúde & $15,84(2,41)$ & $0,14^{*}$ \\
\hline Total & $89,61(10,15)$ & $0,21^{*}$ \\
\hline
\end{tabular}

Nota. $N=436$.

${ }^{*} p<0,05 .{ }^{* *} p<0,01$.

Os dados das análises de regressão são apresentados na Tabela 2. O modelo de predição para a autoeficácia para a escolha profissional indicou que, no Bloco 1, a variável sociodemográfica sexo (feminino) foi responsável por 3\% da variação do desfecho. Com a inclusão do Bloco 2 (fatores das habilidades sociais), o poder de predição aumentou para $19 \%$ do total da variação da autoeficácia para a escolha profissional. As habilidades sociais de desenvoltura social e de abordagem afetiva predisseram positivamente a autoeficácia para a escolha profissional, sendo que a desenvoltura social teve maior peso. O modelo final, que explicou $26 \%$ da variabilidade da autoeficácia para a escolha profissional, incluiu, por ordem de importância, as variáveis habilidades sociais de desenvoltura social, expectativas de futuro em relação ao trabalho e à educação, o sexo (feminino) e as habilidades sociais de abordagem afetiva. 
Tabela 2 - Regressão Hierárquica para a Predição da Autoeficácia para a Escolha Profissional

\begin{tabular}{|c|c|c|c|c|c|}
\hline Preditores & $B(E P)$ & B & $95 \%$ IC & $R^{2}$ & $\Delta R^{2}$ \\
\hline \multicolumn{6}{|l|}{ Bloco 1 - Demográfica } \\
\hline Sexo & $6,69(1,79)$ & $0,17^{*}$ & {$[3,16 ; 10,23]$} & & \\
\hline$F_{(1,43)}$ variação $=13,88^{*}$ & & & & 0,03 & 0,03 \\
\hline \multicolumn{6}{|c|}{ Bloco 2 - Habilidades Sociais } \\
\hline Empatia & $0,20(0,20)$ & 0,06 & {$[-0,19 ; 0,61]$} & & \\
\hline Autocontrole & $-0,23(0,15)$ & $-0,07$ & {$[-0,52 ; 0,07]$} & & \\
\hline Assertividade & $0,15(0,20)$ & 0,04 & {$[-0,25 ; 0,56]$} & & \\
\hline Desenvoltura social & $1,60(0,27)$ & $0,32^{*}+$ & {$[1,07 ; 2,14]$} & & \\
\hline Civilidade & $0,94(0,30)$ & 0,02 & {$[-0,50 ; 0,69]$} & & \\
\hline Abordagem afetiva & $0,56(0,23)$ & $0,12^{*}+$ & {$[0,10 ; 1,01]$} & & \\
\hline$F_{(7,42)}$ variação $=15,66^{* *}$ & & & & 0,19 & 0,17 \\
\hline \multicolumn{6}{|c|}{ Bloco 3 - Expectativas de Futuro } \\
\hline Trabalho/educação & $1,54(0,27)$ & $0,28^{*}$ & {$[1,01 ; 2,06]$} & & \\
\hline Criança/familia & $-0,10(0,37)$ & $-0,01$ & {$[-0,82 ; 0,63]$} & & \\
\hline Casamento & $-0,15(0,37)$ & $-0,02$ & {$[-0,88 ; 0,58]$} & & \\
\hline Igreja & $-0,02(0,26)$ & $-0,01$ & {$[-0,52 ; 0,49]$} & & \\
\hline Saúde & $0,16(0,39)$ & 0,02 & {$[-0,58 ; 0,92]$} & & \\
\hline$F_{(23,29)}$ variação $=13,68^{* *}$ & & & & 0,26 & 0,08 \\
\hline
\end{tabular}

Nota. $N=436 . B$ = coeficiente de regressão não padronizado; EP = Erro Padrão do coeficiente de regressão não padronizado; $\beta$ = coeficiente de regressão padronizado; $I C$ = Intervalo de Confiança; $R^{2}=$ coeficiente de determinação ajustado; $\Delta R^{2}$ = alteração coeficiente de determinação com a entrada dos blocos. Sexo ( 1 = feminino; $0=$ masculino); + Preditores significativos que se mantiveram no modelo final.

${ }^{*} p<0,05 .{ }^{* *} p<0,01$.

\section{Discussão}

O estudo investigou em que medida o sexo, as habilidades sociais e as expectativas de futuro predizem a autoeficácia para a escolha profissional de estudantes do ensino médio. Os resultados das análises de correlação indicaram que estudantes com habilidades sociais apresentavam maiores niveis de crenças de autoeficácia para a escolha profissional, indo ao encontro da literatura (Erozkan, 2013, Harrel et al., 2009; Leme et al., 2016). Os resultados evidenciaram que os alunos com mais expectativas quanto ao futuro tinham mais crenças de autoeficácia para a escotha profissional. Tais resultados são corroborados pelos estudos de Walker e Tracey (2012) e Jung et al. (2015) que demonstraram que estudantes com expectativas positivas em relação ao futuro apresentavam crenças elevadas de autoeficácia para a escolha profissional.

O modelo de regressão indicou que ser adolescente do sexo feminino foi associado a maiores niveis de autoeficácia para a escolha profissional, indo ao encontro de outros estudos (Ambiel \& Noronha, 2014; Guerreiro-Casanova et al., 2015: Noronha et al., 2013). Identifica-se um aumento no número de mulheres no mercado de trabalho nas últimas décadas. Além disso, elas têm uma média maior de anos de estudo quando comparadas aos homens (Oliveira et al., 2016). É possivel que mudanças socioculturais contribuam para 
que as estudantes percebam maior capacidade de escolher a profissão. De acordo com Bandura (2006), as experiências vicariantes são fontes de autoeficácia que possibilitam a aprendizagem a partir da observação das vivências do outro. Diante disso, pode-se inferir que a inserção de figuras femininas em diversos contextos (politico, econômico e social) pode influenciar as crenças de autoeficácia das adolescentes, fazendo com que elas acreditem cada vez mais no seu potencial de assumir esses espaços, outrora ocupados exclusivamente por homens. Contudo, as mulheres brasileiras continuam ainda enfrentando muitas desigualdades quando se trata de trabalho (Oliveira et al., 2016). Assim, embora as mulheres estejam em maioria no ensino médio e superior, continuam a ter taxas de desemprego mais elevadas do que os homens, a desempenhar menos cargos de chefia ou direção e a usufruir de salários mais baixos, mesmo quando desempenham funções semelhantes aos homens (Oliveira et al., 2016).

As análises indicaram que as habilidades sociais desenvoltura social e abordagem afetiva predisseram positivamente as crenças de autoeficácia para a escolha profissional dos adolescentes. Esses dados são compativeis com a literatura da área que afirma que as habilidades sociais contribuem para aumentar as crenças de autoeficácia (Erozkan, 2013; Harrel et al., 2009; Leme et al., 2016). As habilidades sociais de abordagem afetiva compreendem estabelecer contato para relações de amizade, ingressar em grupos na escola ou no trabalho ou, ainda, manter contato e conversação para relações de intimidade (Del Prette \& Del Prette, 2009). Já as habilidades sociais de desenvoltura social consistem em se expor no grupo em apresentações de trabalho, exposição social e conversação geral, manter conversas sobre sexo com os pais e conversar com pessoas de autoridade (Del Prette \& Del Prette, 2009). A aptidão para desempenhar com êxito tarefas desse tipo pode melhorar a interação dos adolescentes com as pessoas a sua volta. Diante disso, os resultados deste estudo sugerem que, quanto mais elaborado for o repertório de habilidades sociais dos adolescentes, maior a probabilidade de eles terem experiências prévias de sucesso nas relações interpessoais e, consequentemente, desenvolver ou fortalecer mais crenças de autoeficácia (Erozkan, 2013; Harrel et al., 2009; Leme et al., 2016). Além disso, existem fatores da autoeficácia para a escolha profissional que podem ser otimizados quando os adolescentes apresentam boa capacidade de interação, como, por exemplo, a autoeficácia para coleta de informações profissionais práticas que demandam orientação de profissionais que atuam na área (Noronha et al., 2013).

De fato, Bandura (2006) defende que as experiências pessoais têm um grande impacto sobre a autoeficácia. Segundo o autor, as vivências prévias de sucesso sustentam a capacidade da pessoa em realizar determinadas tarefas, levando ao desenvolvimento ou ao fortalecimento das crenças de autoeficácia, que posteriormente deverão influenciar na motivação e na persistência para se engajar em atividades do mesmo domínio (Bandura, 2006). Porém, quando o individuo enfrenta fracasso repetido diante de determinadas tarefas, tais experiências podem enfraquecer as apreciações sobre sua eficácia pessoal, interferindo na consecução das atividades (Bandura, 2006).

Com a inserção dos fatores das expectativas de futuro, os resultados indicaram que somente o fator trabalho e educação foi preditor positivo significativo das crenças de autoeficácia para a escolha profissional. Esse resultado era esperado pois, de acordo com a literatura, expectativas positivas em relação ao futuro podem fortalecer as crenças de autoeficácia (Jung et al., 2015; Tsuzuki, 2015: Walker \& Tracey, 2012). Walker e Tracey (2012) propuseram uma associação positiva entre a perspectiva de tempo futuro e a autoeficácia para a escolha profissional, tendo em vista que, para tomar uma decisão, é imprescindivel que a mesma seja considerada valiosa e que comportamentos e objetivos sejam ligados a essa decisão (percepção da instrumentalidade). Dessa forma, os adolescentes que têm mais expectativas de futuro possuem maior facilidade em tomar as medidas necessárias para alcançar a profissão desejada. A partir desse modelo, pode-se supor 
que, fortalecendo o valor dos comportamentos futuros (valência) e a relação desses comportamentos com o alcance dos objetivos (instrumentalidade), é possivel fornecer uma base para as crenças de autoeficácia. Se o indivíduo não percebe a importância das metas ou a ligação das ações atuais com os objetivos futuros, é improvável que desenvolva as habilidades necessárias para executar os comportamentos e, assim, a necessária autoeficácia (Jung et al., 2015; Tsuzuki, 2015; Walker \& Tracey, 2012). Desse modo, os adolescentes que têm boas expectativas com relação ao trabalho e à educação podem investir nessas áreas e, consequentemente, em tarefas relacionadas à escolha da profissão, aumentando, assim, suas crenças de autoeficácia.

\section{Considerações finais}

À luz da Teoria Social Cognitiva (TCS), o presente estudo contribuiu para a maior compreensão de fatores de proteção no processo de transição para a vida adulta, a saber, as crenças de autoeficácia para a escolha profissional, as expectativas de futuro (ambas compreendidas como fatores pessoais) e as habilidades sociais (fatores comportamentais). Para os profissionais que trabalham com adolescentes, sobretudo no contexto escolar, um maior entendimento sobre a relação dessas variáveis pode auxiliar na elaboração de intervenções que visem o desenvolvimento integral dos estudantes. A escola, que é parte da rede de proteção, nem sempre constitui um ambiente favorável para que os alunos desenvolvam boas expectativas acerca do futuro ou que mesmo permanecem na instituição, exigindo assim uma revisão de seus processos educacionais.

Ainda que esta pesquisa tenha pontos fortes, identifica-se limitações. Em primeiro lugar a impossibilidade de generalização dos dados tendo em vista o fato da amostra ter sido constituída por estudantes do estado do Rio de Janeiro, sendo necessária a contextualização dos dados. Por fim, o modelo de predição sinalizou que $26 \%$ da variabilidade das crenças de autoeficácia para a escolha profissional foi explicado pelas variáveis incluídas no modelo. Esse coeficiente de determi- nação é considerado pequeno, o que sugere que outras variáveis podem influenciar tais crenças, devendo ser investigadas em futuros estudos.

Consideradas as limitações do presente estudo, sugere-se que trabalhos posteriores podem ampliar a amostra por meio da coleta de dados com adolescentes de outras regiões; investigar outras variáveis que podem afetar a autoeficácia para a escolha profissional, tais como a percepção de apoio social e as habilidades sociais de pais e professores; propor e avaliar programas de intervenção com os adolescentes, suas familias e professores sobre as influências das habilidades sociais, expectativas de futuro e estereótipos de gênero sobre a escolha profissional durante o ensino médio.

\section{Referências}

Ambiel, R. A., \& Noronha, A. P. P. (2012). Autoeficácia para escolha profissional: Teoria, pesquisas e avaliação. Psicologia em Pesquisa, 6(2), 171-178. https://doi. org/10.5327/Z1982-12472012000200010

Ambiel, R. A. M., \& Noronha, A. P. P. (2014). Escala de autoeficácia para escolha profissional (EAE-EP): Manual técnico (2. ed.). Casa do Psicólogo.

Arnett, J. J. (2016). Does emerging adulthood theory apply across social classes? National data on a persistent wuestion. Emerging Adulthood, 4(4), 227-235. https://doi.org/10.1177/2167696815613000

Azzi, R. G., Vieira, D. A., laochite, R. T., Ferreira, L. C. M., \& Guerreiro-Casanova, D. C. (2014). Crenças de eficácia pessoale coletiva. In R. G. Azzi \& D. A. Vieira (Eds.), Crenças de eficácia em contexto educativo (pp. 15-40). Casa do Psicólogo.

Bandura, A. (1986). Social foundations of thought and action: A social cognitive theory. Prentice- Hall, Inc.

Bandura, A. (2006). Toward a psychology of human agency. Perspectives on Psychological Science, 1, 164. https://doi.org/10.1111/j.17456916.2006.00011.X

Berni, V. L. \& Roso, A. (2014). A adolescência na perspectiva da psicologia social critica. Psicologia \& Sociedade, 26(1), 126-136. https://doi.org/10.1590/S010271822014000100014

Bolat, N., \& Odaci, H. (2017). High school final year students' career decision-making self-efficacy, attachment styles and gender role orientations. Current Psychology, 36(2), 252-259. https://doi.org/10.1007/ \$12144-016-9409-3

Braga, M. J., \& Xavier, F. P. (2016). Transição para o ensino superior: Aspiração dos alunos do ensino médio de uma escola pública. Educar em Revista, (62), 245-259. https://doi.org/10.1590/0104-4060.46817

Bryman, A, \& Cramer, D. (2003). Análise de dados em ciências sociais (3. ed.). Celta. 
Chiesa, R., Massei, F., \& Guglielmi, D. (2016). Career decision-making self-efficacy change in italian high school students. Journal of Counsenling \& Development, 94(2), 210-224. https://doi.org/10.1002/jcad.12077

Dantas, M. A., \& Azzi, R. G. (2018). Relato de uma experiência de intervenção na perspectiva da teoria social cognitiva de carreira. Temas em Psicologia, 26(2), 735749. https://doi.org/10.9788/TP2018.2-08Pt

Del Prette, Z. A. P., \& Del Prette, A. (2009). Inventário de Habilidades Sociais para adolescentes. (IHSA - Del-Prette): Manual de aplicação, apuração e interpretação. Casa do Psicólogo.

Del Prette, Z. A. P., \& Del Prette, A. (2010). Habilidades Sociais e análise do comportamento: Proximidade histórica e atualidades. Perspectivas em Análise do Comportamento, 1(2), 38-49. http://pepsic.bvsalud.org/ $\mathrm{pdf/pac/v1n2/v1n2a04.pdf}$

Dutra-Thomé, L., Koller, S. H., McWhirter, E. H., \& McWhirter, B. (2015). Application of the Future Expectation Scale for Adolescents (FESA) in Brazil. Psicologia: Reflexão e Critica, 28(2), 331-339. https://doi.org/10.1590/16787153.201528213

Dutra-Thomé, L., \& Koller, S. H. (2017). Brazilian version of the Inventory of the Dimensions of Emerging Adulthood: Investigating the current transition to adulthood. Temas em Psicologia, 25(3), 901-912. https://doi.org/10.9788/ TP2017.3-01

Erozkan, A. (2013). The effect of communication skills and interpersonal problem solving skills on social self-efficacy. Educational Sciences: The Theory \& Practice, 13(2), 739-745. https://files.eric.ed.gov/fulltext/ EJ1017303.pdf

Guerreiro-Casanova, D. C., Dantas, M. A., \& Azzi, R. G. (2015). Aspectos pessoais e escolares associados à autoeficácia acadêmica no Ensino Médio. Psicologia Ensino e Formação, 6(1), 1-18. http://pepsic.bvsalud. org/pdf/pef/v6n1/v6n1ao6.pdf

Hackett, G. \& Betz, N. E. (1981). A self-efficacy approach to the career development of women. Journal of Vocational Behavior, 18(1), 326-336. https://doi. org/10.1016/0001-8791(81)90019-1

Harrel, M. W., Mercer, S. H., \& DeRosier, M. E. (2009). Improving the social-behavior adjustment of adolescents: The effectiveness of a social skills group intervention. Journal of Child and Family Study, 18(1), 378-387. https:// doi.org/10.1007/s10826-008-9241-y

Husman, J., \& Lens, W. (1999). The role of the future in student motivation. Educational Psychologist, 34(1), 113-125. https://doi.org/10.1207/s15326985ep3402_4

Instituto Nacional de Estudos e Pesquisas Educacionais Anisio Teixeira. (2017). Censo Escolar 2017: Notas estatisticas. Ministério da Educação. https://drive.google.com/ file/d/1ul80ptGdTzory5Jom-TvvSzILCrXmWeE/view

Jung, H., Park, I. J., \& Rie, J. (2015). Future time perspective and career decisions: The moderating effects of affect spin. Journal of Vocational Behavior, 89(1), 46-55. https://doi.org/10.1016/j.jvb.2015.04.010
Leme, V. B. R., Campos, J. R., Coimbra, S., Del Prette, A., \& Del Prette, Z. A. P. (2016). Social skills inventory for adolescents: Evidence of construct validity and reliability. Psico, 47(3), 171-178. https://doi.org/10.15448/19808623.2016.3.20942

Leme, V. B. R., Fernandes, L. M., Jovarini, N. V., El Achkar, A. M., \& Del Prette, Z. A. P. (2016). Social Skills Program for Adolescents in Vulnerable Social Contexts. Psico-USF, 21(3). https://doi.org/10.1590/1413-82712016210313

Lent, R. W., Brow, S. D., \& Hackett, G. (1994). Towards a unifying social cognitive theory of career and academic interests, choice and performance. Journal of Vocational Behavior, 45(1), 79-122. https://doi.org/10.1006/ jvbe.1994.1027

McWhirter, E. H., \& McWhirter, B. T. (2008). Adolescent future expectations of work, education, family, and community: Development of a new measure. Youth \& Society, 20(10), 1-21. https://doi. org/10.1177/0044118X08314257

Moreira, T. C., Ambiel, R. A. M., \& Nunes, M. F. O. (2018). Escala de fontes de autoeficácia para escoha professional: Construção e estudos psicométricos iniciais. Temas em Psicologia, 26(1), 47-60. https://doi.org/10.9788/ TP2018.1-03Pt

Noronha, A. P. P., Freitas, P. C. S., Piovezan, N. M., \& Joly, M. C. R. A. (2013). Afetos positivos e negativos e autoeficácia em jovens do ensino médio. Revista de Psicología, 15(1), 9-21. http://revistas.ucv.edu.pe/index. php/R_PSI/article/view/206/117

Nurmi, J. E. (1991). How do adolescents see their future? A review of the development of future orientation and planning. Developmental Review, 11(1), 1-59. https://doi. org/10.1016/0273-2297(91)90002-6

Oliveira, T. D., Golgher, A. B., \& \& Loureiro, P. M (2016). Trajetórias de local de moradia, estudo e trabalho dos jovens brasileiros entre 2003 e 2011: Uma análise de entropia. Revista Brasileira de Estudos Populacionais, 33(1), 31-51. https://doi.org/10.20947/S0102-309820160003

Ponciano, E. L. T., \& Féres-Carneiro, T. (2014). Relação pais-filhos na transição para a vida adulta, autonomia e relativização da hierarquia. Psicologia Reflexão e Critica, 27(2), 388-397. https://doi.org/10.1590/16787153.201427220

Prisco, A. P. K., Martins, C. R., \& Nunes, M. F. O. (2013). Estudos sobre autoeficácia aplicada ao desenvolvimento de carreira no Brasil: Uma revisão. Revista Brasileira de Orientação Profissional, 14(1), 111-118. http://pepsic. bvsalud.org/pdf/rbop/v14n1/11.pdf

Sovet, L., \& Metz, A. J. (2014). Parenting styles and career decision-making among French and Korean adolescents. Journal of Vocational Behavior, 84(3), 345-355. https://doi.org/10.1016/j.jvb.2014.02.002

Taylor, K. M., \& Betz, N. E. (1983). Applications of self-eficacy theory to the understanding and treatment of career indecision. Journal of Vocational Behavior, 22, 63-81. https://doi.org/1 0.1016/0001-8791(83)90006-4 
Tsuzuki, M. (2015). Examination about the effects of future career choice on time perspective in japanese high school students. The Spanish Journal of Psychology, 18(17), 1-6. https://doi.org/10.1017/sjp.2015.21

Ventura, C. D., \& Noronha, A. P. P. (2014). Autoeficácia para escolha profissional, suporte familiar e estilos parentais em adolescentes. Avaliação Psicológica, 13(3), 317-324. http://pepsic.bvsalud.org/pdf/avp/ v13n3/v13n3a03.pdf

Walker, T. L., \& Tracey, T. J. (2012). The role of future time perspective in career decision-making. Journal of Vocational Behavior, 81(2), 150-158. https://doi.or$\mathrm{g} / 10.1016 / \mathrm{j} . j \mathrm{jb} .2012 .06 .002$

\section{Gisele Aparecida de Morais}

Mestre em Psicologia pela Universidade Salgado de Oliveira (UNIVERSO), em Niterói, RJ, Brasil; psicóloga no Instituto Federal de Educação Ciência e Tecnologia Fluminense, Campus Itaperuna (IFF), em Itaperuna, RJ, Brasil.

\section{Vanessa Barbosa Romera Leme}

Doutora em Psicologia pela Universidade de São Paulo (USP-FFCLRP), em Ribeirão Preto, SP, Brasil; professora no Programa de Pós-Graduação em Psicologia Social na Universidade do Estado do Rio de Janeiro (UERJ), no Rio de Janeiro, RJ, Brasil.

\section{Amanda Oliveira Falcão}

Mestre em Psicologia pela Universidade Salgado de Oliveira (UNIVERSO), em Niterói, RJ, Brasil; professora no Instituto Superior de Teologia Aplicada (UNINTA), em Sobral, CE, Brasil.

\section{Camila de Sousa Pereira-Guizzo}

Doutora em Educação Especial pela Universidade Federal de São Carlos (UFSCar), em São Carlos, SP, Brasil; professora no Centro Universitário Serviço Nacional de Aprendizagem Industrial (SENAI CIMATEC), em Salvador, BA, Brasil.

\section{Endereço para correspondência}

Gisele Aparecida de Morais

Rua Sergio Dias Pecly, 20/301

28300-000

Itaperuna, RJ, Brasil 